\title{
High rate of complete responses to immune checkpoint inhibitors in patients with relapsed or refractory Hodgkin lymphoma previously exposed to epigenetic therapy
}

\author{
Lorenzo Falchi', Ahmed Sawas', Changchun Deng ${ }^{1}$, Jennifer E. Amenguall', Donald S. Colbourn', \\ Emily A. Lichtenstein ${ }^{1}$, Karen A. Khan ${ }^{1}$, Lawrence H. Schwartz ${ }^{2}$ and Owen A. O'Connor ${ }^{\text {* }}$
}

\begin{abstract}
Options for patients with relapsed or refractory $(\mathrm{R} / \mathrm{R})$ classical Hodgkin lymphoma $(\mathrm{CHL})$ after brentuximab vedotin (Bv) and autologous stem cell transplantation (ASCT) are limited. Immune checkpoint inhibitors (ICI) are active in this population but rarely induce complete response (CR). Ten patients with R/R CHL after ASCT and Bv received pembrolizumab $(n=8)$ or nivolumab $(n=2)$. Five had been previously exposed to 5 -azacitidine on a phase 1 study. Among nine evaluable patients, seven (78\%) achieved $C R$, one partial response, and one reduction of tumor burden. All five patients who had received 5-azacitidine prior to $\mathrm{ICl}$ achieved $\mathrm{CR}$, while only two of four who did not receive prior 5-azacitidine achieved CR. At a median follow-up of 9.9 months [0.5-14.3], eight patients are alive and five are still receiving treatment. We documented an unprecedented $C R$ rate after $I C l$ in patients with $R / R \mathrm{cHL}$. We hypothesize that hypomethylating agents might have an immune priming effect and enhance the efficacy of ICl.
\end{abstract}

Keywords: Hodgkin lymphoma, Pembrolizumab, Nivolumab, Azacitidine, Epigenetic therapy

\section{To the editor}

Patients with classical Hodgkin lymphoma (cHL) refractory to, or relapsed after, autologous stem cell transplantation (ASCT) and brentuximab vedotin (Bv) have limited treatment options and represent an unmet medical need [1].

The immune checkpoint receptor programmed death (PD)-1 is expressed on T cells and causes reversible anergy when engaged by its ligands after antigen recognition by the T cell receptor [2]. Expression of PD-ligand (L) 1 or PD-L2 is utilized by tumors to escape immune surveillance [3]. cHL exhibits a genetically determined overexpression of PD-L1/PD-L2 due to recurrent amplification of 9q24.1 [4], and thus may be exquisitely sensitive to PD-1 blockade. The anti-PD-1 monoclonal

\footnotetext{
* Correspondence: o02130@cumc.columbia.edu

${ }^{1}$ Center for Lymphoid Malignancies, Division of Hematology/Oncology, Department of Medicine, Columbia University Medical Center, 51 West 51st Street, Suite 200, New York, NY 10019, USA

Full list of author information is available at the end of the article
}

antibodies nivolumab and pembrolizumab, two immune checkpoint inhibitors (ICI), are highly active in patients with relapsed or refractory $(\mathrm{R} / \mathrm{R}) \mathrm{cHL}$ but induced complete response $(\mathrm{CR})$ only in a minority of them $[5,6]$. The hypomethylating agent (HMA) 5-azacitidine was shown to induce expression of retroviral genes in tumor cells and trigger a $\mathrm{T}$ cell-mediated response, thus potentially synergizing with ICI [7]. We report our experience with ICI in ten patients with $\mathrm{R} / \mathrm{R} \mathrm{cHL}$, five of whom were previously exposed to 5 -azacitidine.

Eight patients received pembrolizumab $(2 \mathrm{mg} / \mathrm{kg}$ every 3 weeks) and two nivolumab (3 mg/kg every 2 weeks). Response was evaluated with fluorodeoxyglucose-positron emission tomography/computerized tomography (PET/ CT) according to the 2014 Lugano criteria [8]. Patients with new or persistent lesions were allowed to continue on therapy if they had disease and/or symptom control, without unacceptable toxicity. 
The median number of prior treatments was 11 [3-16] and $80 \%$ of patients had received $\geq 7$ lines of therapy. All patients had received ASCT and $\mathrm{Bv}$ (Additional file 1: Table S1). Five patients had been previously treated with 5 -azacitidine in combination with romidepsin within a phase 1 clinical trial (NCT01998035) (Additional file 2: Table S2). Median duration of 5-azacitidine therapy was 3 months [2-16]. Three patients received it immediately prior to ICI, the other two within 14 months of initiating ICI (Fig. 1).

The median treatment duration was 25 weeks [2-54]. Two treatment delays lasting $>1$ week were due to lack of insurance coverage and diagnosis of myelodysplastic syndrome (MDS) (Fig. 2). There were six grade $\geq 3$ adverse events $(\mathrm{AE})$ : one patient had an infusion reaction, one had thrombocytopenia and was later diagnosed with MDS, and another developed chronic myelomonocytic leukemia (CMML) right after treatment initiation. The latter two patients had been exposed to radiation and alkylating agents and developed acute myeloid leukemia (AML), fatal in one case. One patient with chronic kidney disease developed a non-steroidal anti-inflammatory drug-related interstitial nephritis, in resolution at the time of pembrolizumab initiation. One patient developed hypotension, hypoxia and bilateral pulmonary infiltrates after the first infusion and died 2 weeks later of multiorgan failure, despite broad-spectrum antibiotics and highdose steroids. This patient had pre-existent depressed left ventricular ejection fraction (20\%), recurring pericardial effusion, post-radiation fibrosis and bronchiectasis, and recent pneumonia. Grade 1-2 AE are summarized in Additional file 3: Table S3.

Nine patients were evaluable for response. Seven (78\%) achieved CR and one partial response. One patient had a significant reduction of all tumor sites and developed a new liver lesion. All five patients who had been exposed to 5-azacitidine containing therapy achieved a CR, whereas only two of the four who did not receive prior 5-azacitidine achieved CR (Fig. 2). After a median follow-up of 9.9 months [0.5-14.3] eight patients were alive and five were still receiving treatment after 23, 39, 48,48 , and 54 weeks. The patient with a new liver lesion had stable disease, no new symptoms, and no significant toxicity, thus continued to receive ICI therapy. Three patients discontinued therapy: one transitioned to alloSCT while in CR after 9 weeks of therapy and remains in remission 1 year later; one discontinued while in CR after 17 weeks, due to transformation of CMML into AML;

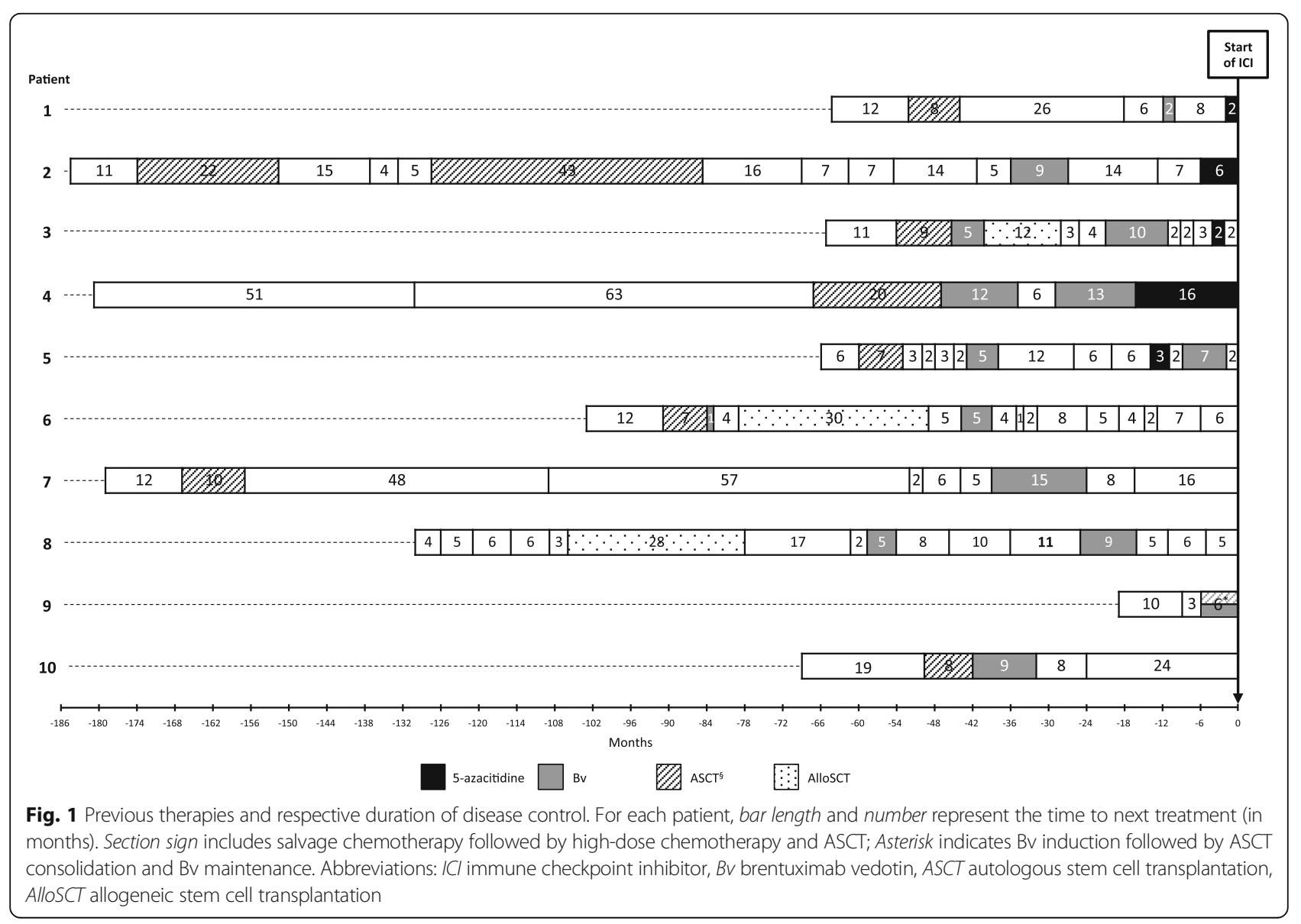




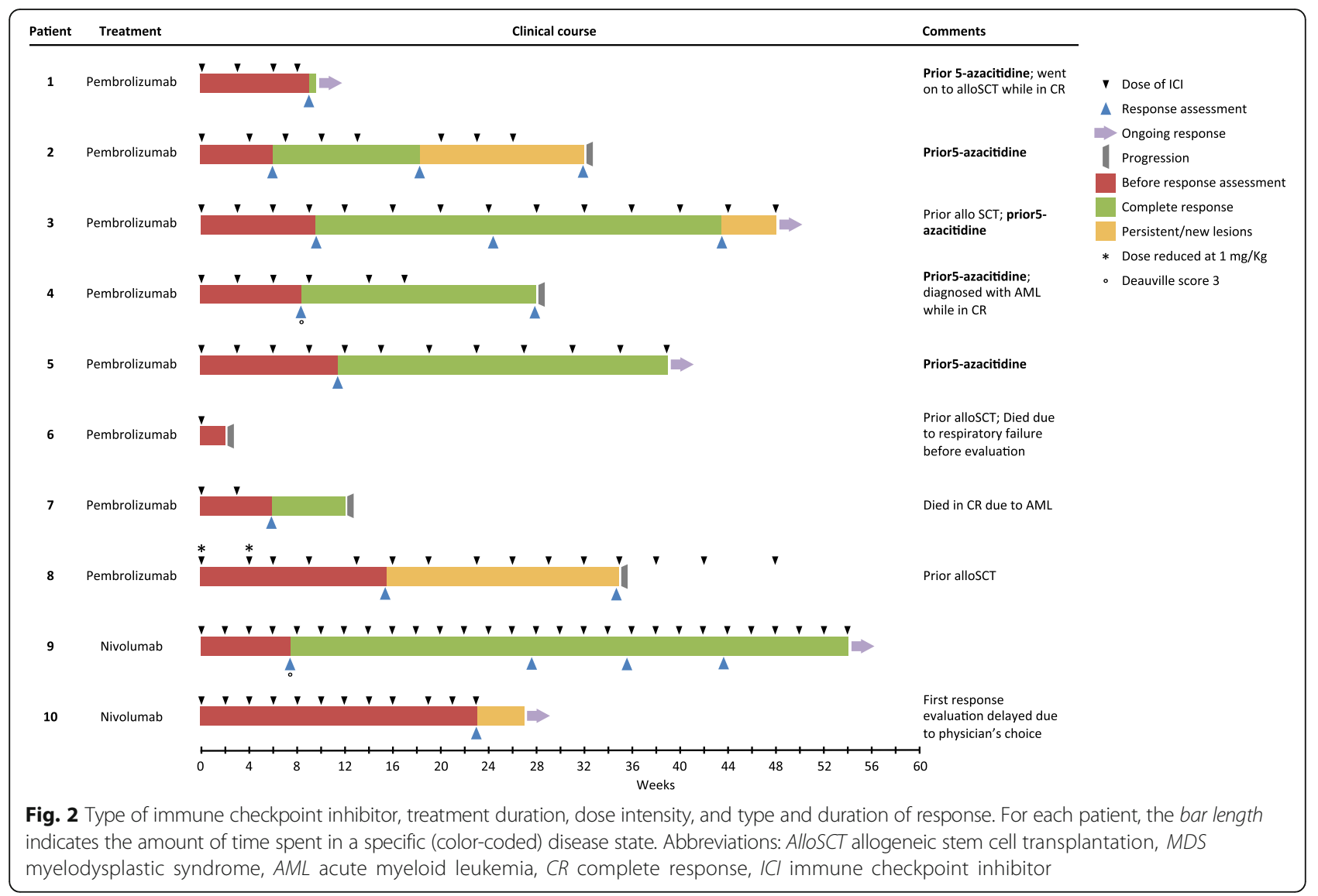

and the third patient discontinued treatment due to confirmed progression and lack of clinical benefit after 32 weeks (Fig. 2).

To the best of our knowledge, this is the first report to suggest clinical synergy between epigenetic therapy and ICI in patients with cHL. In this very heavily pre-treated cohort, ICI showed impressive clinical efficacy with a CR rate (CRR) of nearly $80 \%$. In phase 1 trials in patients with $\mathrm{R} / \mathrm{R} \mathrm{cHL}$, nivolumab $(n=23)$ induced an overall response rate (ORR) and CRR of $87 \%$ and $17 \%$, respectively [5], and pembrolizumab $(n=31)$, at a dose of $10 \mathrm{mg} / \mathrm{kg}$, an ORR of $65 \%$ and CRR of $16 \%$ [6]. With the limitations of comparing separate, small series, our cohort appears to include more extensively pre-treated patients (Additional file 1: Table S1 and $[5,6]$ ). Yet, most of them achieved CR early after treatment.

All five patients previously exposed to 5 -azacitidine obtained PET/CT-negative CR. Recent studies suggested that HMA can induce up-regulation of endogenous retroviral genes in tumor cells. Endocellular sensors of viral doublestrand RNA then trigger an interferon- $\beta$-mediated $\mathrm{T}$ cell response. Moreover, in a pre-clinical melanoma model, 5azacitidine was found to sensitize cells to ipilimumab, another ICI [7]. This agent may, therefore, have a "priming" effect on the immune system and maximize the response to ICI. The impact of 5-azacitidine might be harder to assess in patient N. 5 , as he received the drug about 1 year prior to starting ICI. Unlike 5-azacitidine, histone deacetylase inhibitors have not been shown to synergize with ICI. However, because 5-azacitidine was administered in combination with romidepsin in our patients, we cannot exclude that additional romidepsin-mediated modulation of gene expression might have further potentiated the effects of ICI, thus contributing to the high CRR. Other limitations of our retrospective study include the small sample size, the retrospective nature of the study, the heterogeneous duration and timing of HMA therapy, and the use of different ICI. Nevertheless, the uniquely high CRR cannot be explained by ICI therapy alone and may be in part due to synergy with HMA. The combination of epigenetic therapy and PD-1 blockade is being tested prospectively at our institution.

\section{Additional files}

Additional file 1: Table S1. Characteristics of the patients included in the present report and the studies from Ansell and colleagues and Armand and colleagues. (DOCX $90 \mathrm{~kb}$ )

Additional file 2: Table S2. Treatment schedule of combined 5azacitidine and romidepsin (phase 1 study, NCT01998035). (DOCX 57 kb) 
Additional file 3: Table S3. Adverse events observed during immune checkpoint inhibitor therapy. (DOCX $15 \mathrm{~kb}$ )

\section{Abbreviations}

AE: Adverse event; AML: Acute myeloid leukemia; ASCT: Autologous stem cell transplantation; Bv: Brentuximab vedotin; CHL: Classical Hodgkin Iymphoma; CMML: Chronic myelomonocytic leukemia; CR: Complete response; CRR: Complete response rate; HMA: Hypomethylating agent; ICl: Immune checkpoint inhibitor(s); MDS: Myelodysplastic syndrome; ORR: Overall response rate; PD-1: Programmed death-1; PD-L1, PD-L2: PDligand 1, PD-ligand 2; PET/CT: Fluorodeoxyglucose-positron emission tomography/computerized tomography; R/R: Relapsed or refractory

\section{Acknowledgements}

Not applicable.

\section{Funding}

The authors did not receive support for this study.

\section{Availability of data and materials}

The datasets supporting the conclusions of this article are included within the article (and its additional files).

\section{Authors' contributions}

$\mathrm{LF}$ and $\mathrm{OAO}$ conceived and designed the research study and drafted the manuscript. LF, AS, CD, JEA, DSC, EAL, KAK, LHS, and OAO acquired, analyzed, and interpreted data and helped to draft the manuscript. AS, CD, JEA, DSC, $E A L, K A K, L H S$, and OAO were involved in patient care. All authors read, revised, and approved the manuscript.

\section{Competing interests}

The authors declare that they have no competing interests.

\section{Consent for publication}

Waived under protocol IRB-AAAQ8881.

\section{Ethics approval and consent to participate}

This study was approved by the Columbia University Medical Center Institutional Review Board (protocol number: IRB-AAAQ8881). Waiver of consent to participation in the study was granted.

\section{Author details}

${ }^{1}$ Center for Lymphoid Malignancies, Division of Hematology/Oncology, Department of Medicine, Columbia University Medical Center, 51 West 51st Street, Suite 200, New York, NY 10019, USA. ${ }^{2}$ Department of Radiology, Columbia University Medical Center, 180 Fort Washington Avenue, New York, NY 10032, USA.

Received: 7 October 2016 Accepted: 22 November 2016 Published online: 30 November 2016

\section{References}

1. Gopal AK, Chen R, Smith SE, Ansell SM, Rosenblatt JD, Savage KJ, Connors JM, Engert A, Larsen EK, Chi X, et al. Durable remissions in a pivotal phase 2 study of brentuximab vedotin in relapsed or refractory Hodgkin lymphoma. Blood. 2015;125:1236-43.

2. Keir ME, Butte MJ, Freeman GJ, Sharpe AH. PD-1 and its ligands in tolerance and immunity. Annu Rev Immunol. 2008;26:677-704.

3. Pardoll DM. The blockade of immune checkpoints in cancer immunotherapy. Nat Rev Cancer. 2012;12:252-64.

4. Roemer MG, Advani RH, Ligon AH, Natkunam Y, Redd RA, Homer H, Connelly CF, Sun HH, Daadi SE, Freeman GJ, et al. PD-L1 and PD-L2 genetic alterations define classical Hodgkin lymphoma and predict outcome. J Clin Oncol. 2016; 34(23):2690-7.

5. Ansell SM, Lesokhin AM, Borrello I, Halwani A, Scott EC, Gutierrez M, Schuster SJ, Millenson MM, Cattry D, Freeman GJ, et al. PD-1 blockade with nivolumab in relapsed or refractory Hodgkin's lymphoma. N Engl J Med. 2015;372:311-9.

6. Armand P, Shipp MA, Ribrag V, Michot JM, Zinzani PL, Kuruvilla J, Snyder ES, Ricart AD, Balakumaran A, Rose S, Moskowitz CH. Programmed death-1 blockade with pembrolizumab in patients with classical Hodgkin lymphoma after brentuximab vedotin failure. J Clin Oncol. 2016;34(31):3733-39.

7. Chiappinelli KB, Strissel PL, Desrichard A, Li H, Henke C, Akman B, Hein A, Rote NS, Cope LM, Snyder A, et al. Inhibiting DNA methylation causes an interferon response in cancer via dsRNA including endogenous retroviruses. Cell. 2015;162:974-86.

8. Cheson BD, Fisher RI, Barrington SF, Cavalli F, Schwartz LH, Zucca E, Lister TA, Alliance AL, Lymphoma G, Eastern Cooperative Oncology G, et al. Recommendations for initial evaluation, staging, and response assessment of Hodgkin and non-Hodgkin lymphoma: the Lugano classification. J Clin Oncol. 2014;32:3059-68.

\section{Submit your next manuscript to BioMed Central and we will help you at every step:}

- We accept pre-submission inquiries

- Our selector tool helps you to find the most relevant journal

- We provide round the clock customer support

- Convenient online submission

- Thorough peer review

- Inclusion in PubMed and all major indexing services

- Maximum visibility for your research

Submit your manuscript at www.biomedcentral.com/submit
Biomed Central 